\title{
Study on Newborn Admitted in Nicu with Respiratory Distress
}

\author{
Dr.Antony Kisku ${ }^{1}$, Dr.M.R.Akhouri ${ }^{2}$ \\ ${ }^{1}$ Postgraduate Student, \\ ${ }^{2}$ AssociateProfessorDepartment of Pediatrics,Rajendra Institute of Medical Sciences,Ranchi,Jharkhand,
}

\begin{abstract}
Respiratory Distress in newborn is defined as the presence of any two of the following features:1.Respiratory Rate >60/min.2.Subcostal/Intercostal retractions.3.Expiratory grunt/Groaning. Inaddition, presence of nasal flaring, suprasternal retactions, decreased air entry on auscultation of chest wall will also indicate the presence of respiratory distress. An infant who has an advanced degree of respiratory distress may exhibit additional signs, such as cyanosis, gasping, choking,apnea,stridor. We performed the above study to find out various etiologies and related data to Respiratory Distress in newborns.
\end{abstract}

Keywords: apnea, cyanosis, newborn, respiratory distress, retactions

\section{Introduction}

Respiratory Distress is one of the commonest cause of morbidity and mortality in new born ${ }^{1}$.Newborn child becomes dependent upon its own respiratory system as soon as it separates from the mother's womb. The lung which is solid till the moment of birth must expand promptly. Thus initiation of breathing within few minutes after birth is important foroxygenation and proper functioning of vital organs like brain,heart,kidney etc. Respiratory Distress is caused mainly by the disorders of the respiratory system. The non - respiratory causes are intracranial injury, birthasphyxia, cardiac failure,septicaemia,metabolic disorders and congenital malformations( diaphragmatic hernia, lobar emphysema) ${ }^{2}$.The clinical diagnosis of Respiratory Distress is made when respiratory rate is $>60 / \mathrm{min}$ in quiet resting baby and there is retraction, grunting and nasal flaring, other manifestations include apnea,cyanosis and poor feeding ${ }^{3}$.A history of premature delivery and onset of respiratory distress within six hours of life suggests Hyaline Membrane Disease ${ }^{4}$. A history of premature rupture of membrane and if it is associated with signs of infections in the mother such as foul smelling amniotic fluid, arouses suspicion of bacterial sepsis with intrauterine pneumonia. A history of birth which needed positive pressure ventilation alerts one to the possibility of pneumothorax, so far investigations are concerned, $\mathrm{x}$-ray chest is the minimum investigation that must be done even in the absence of any detectable clinical signs over the chest. Early diagnosis of respiratory distress etiologies and their relevant treatment helps in reducing the mortality and morbidity.

\section{Materials And Methods}

The present study was carried out in the Neonatal Intensive Care Unit ,Rajendrea Institute Of Medical Science(RIMS),Ranchi,Jhrkhand.

\section{Inclusion Criteria}

100 newborns admitted in the NICU,RIMS, RANCHI with the following features were studied ${ }^{5}$ : 1.Respiratory Rate $>60 / \mathrm{min}$. 2.Subcostal Retractions. 3.Intercostal Retractions. 4.Xiphoid Retractions. 5.Suprasternal Retractions. 6.Expiratory grunt. 7.Flaring of alae nasi.

Detailed history including maternal antenatal, natal and post natal and clinical examination was done in each case.

Investigations done:1.Complete blood cell count,HB\%,BAND CELLS COUNT,C-REACTIVE PROTEIN. 2.Gastric aspirate for Meconium staining and polymorphonuclear cells counts were studied in baby born with mother having history of prolonged rupture of membrane(PROM). 3.X-Ray Chest. 4.Cranial Sonography. 5.Examination of CSF,ECG,ECHOCARDIOGRAPHY. 6.Blood culture in appropriate cases to exclude bacterial infections.

\section{Clinical examination was done under two broad heading:}

General ExaminationVitals,anthropometry,head and face, mouth and throat,neck,nail,skin.

Systemic ExaminationCVS,Respiartory system,CNS,Abdominal Exmination,Assesment of gestational age at birth (by MODIFIED BALLARD SCORE) 


\section{Results}

As per the study done following results were obtained which have been documented in various tables and charts mentioned below.

Table 1

Showing Etiology and Percentage of Respiratory Distress In Newborns

\begin{tabular}{|l|l|l|}
\hline ETILOGY & NO. OF PATIENTS & PERCENTAGE \\
\hline Infection & 32 & 32 \\
\hline Transient tachypnea of Newborn & 22 & 22 \\
\hline Birth Asphyxia & 7 & 7 \\
\hline Meconium Aspiration Syndrome & 8 & 8 \\
\hline Hyaline Membrane Disease & 15 & 15 \\
\hline Others & 16 & 16 \\
\hline
\end{tabular}

Histogram 1: Showing Incidence Of Respiratory Distress Among Male And Females

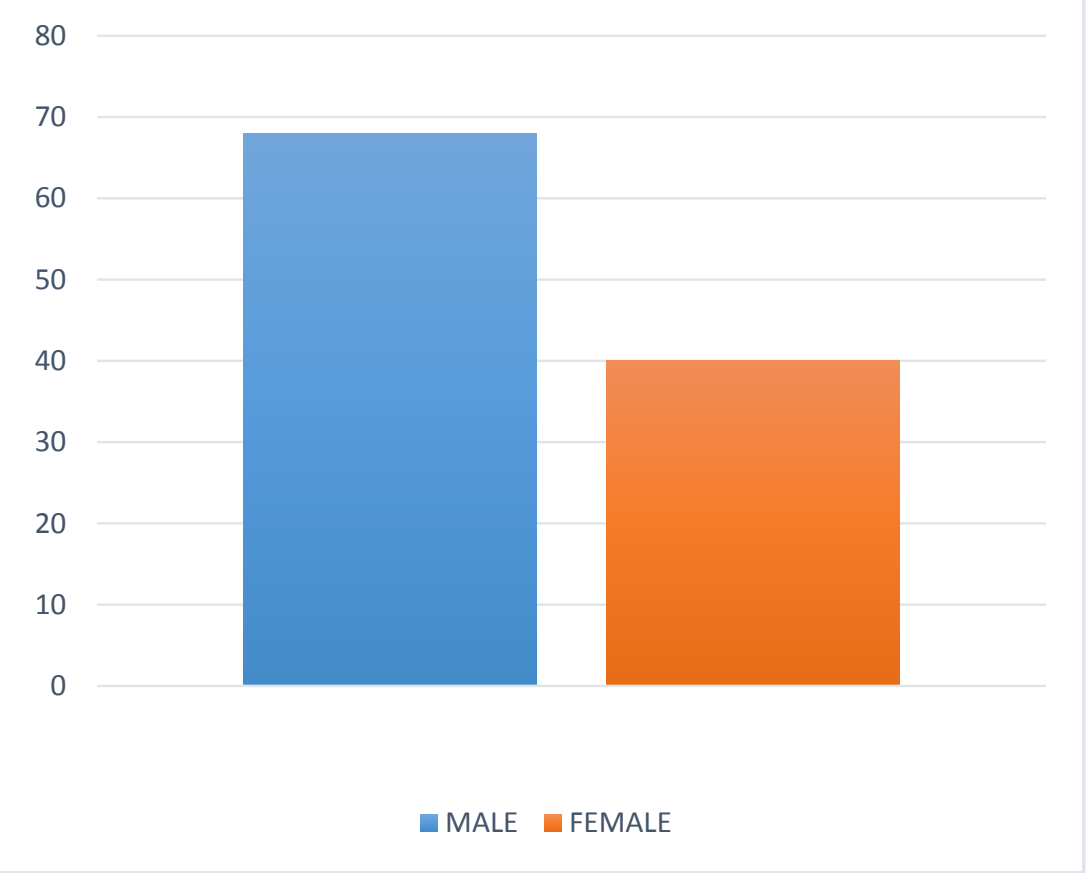

Pie-chart 1ow:Showing distribution of pulmonary and extra pulmonary causes of respiratory distress

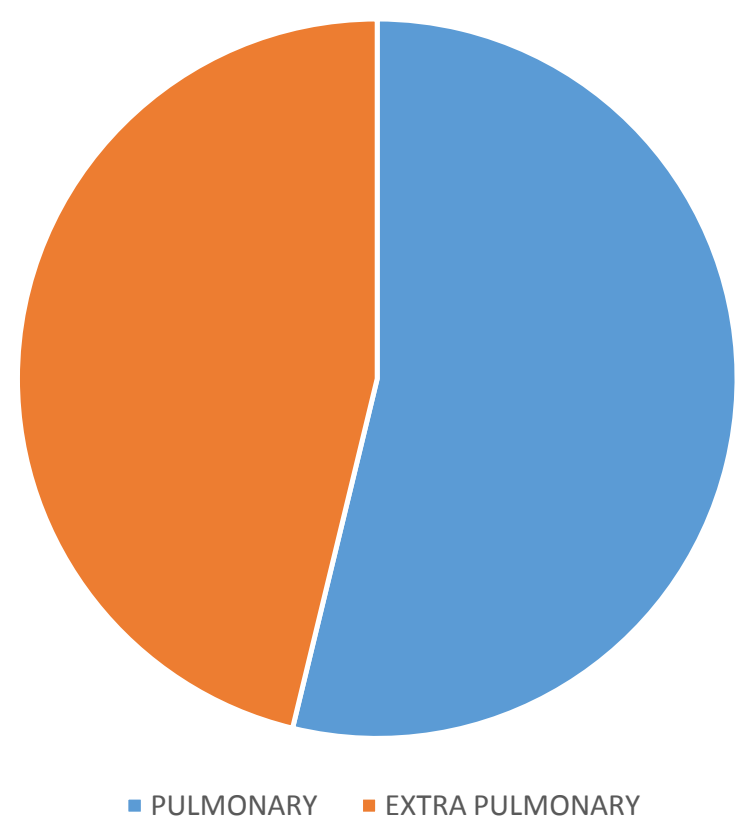


Table2.Showing Incidence Of Respiratory Distress In Term and Pre-Term

\begin{tabular}{|c|c|}
\hline TERM & PRE-TERM \\
\hline 38 & 62 \\
\hline
\end{tabular}

Table 3: Showing variation of Respiratory Distress weight wise

\begin{tabular}{|l|l|}
\hline Birth Weight $(\mathrm{KG})$ & No. Of Cases With Respiratory Distress \\
\hline Up to $1.5 \mathrm{KG}$ & 20 \\
\hline $1.6-2 \mathrm{KG}$ & 15 \\
\hline $2.1-2.5$ & 35 \\
\hline$>2.6$ & 30 \\
\hline & Total $=100$ \\
\hline
\end{tabular}

Table 4: Showing Gestational Age Wise Variation Of Hyaline Membrane Disease

\begin{tabular}{|l|l|l|l|}
\hline$<28$ weeks & $28-32$ weeks & $33-34$ weeks & $>34$ weeks \\
\hline 2 & 9 & 3 & 1 \\
\hline
\end{tabular}

Pie-Chart 2:Showing variation Of Respiratory Distress with Mode OF Delivery Wise:LSCS(35\%) NVD(65\%)

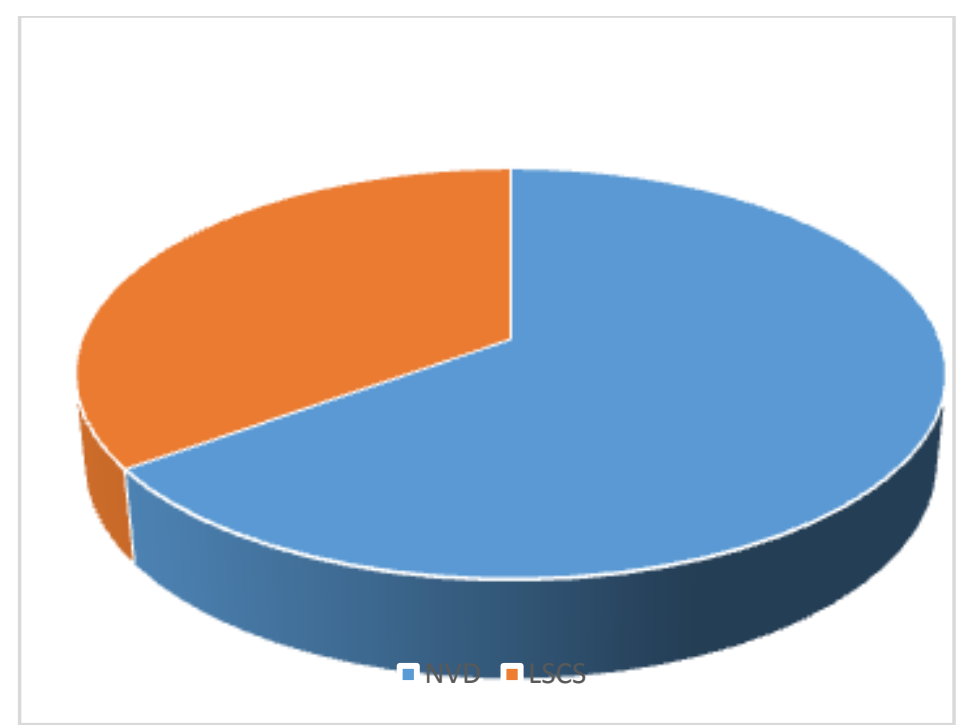

Table-5:Showing Mortality percentages Etiology Wise

\begin{tabular}{|l|l|l|}
\hline Etiology & No. of Deaths & $\%$ Of Mortality \\
\hline Infections & 13 & $40 \%$ \\
\hline Transient Tachypnea Of Newborn & 0 & $0 \%$ \\
\hline Birth Asphyxia & 2 & $28.57 \%$ \\
\hline Meconium Aspiration Syndrome & 2 & $25 \%$ \\
\hline Hyaline Membrane Disease & 6 & $40 \%$ \\
\hline Others & 5 & $31.25 \%$ \\
\hline & Total=28 & Total=28\% \\
\hline
\end{tabular}

Picture below showing subcostal retractions in a newborn with respiratory distress

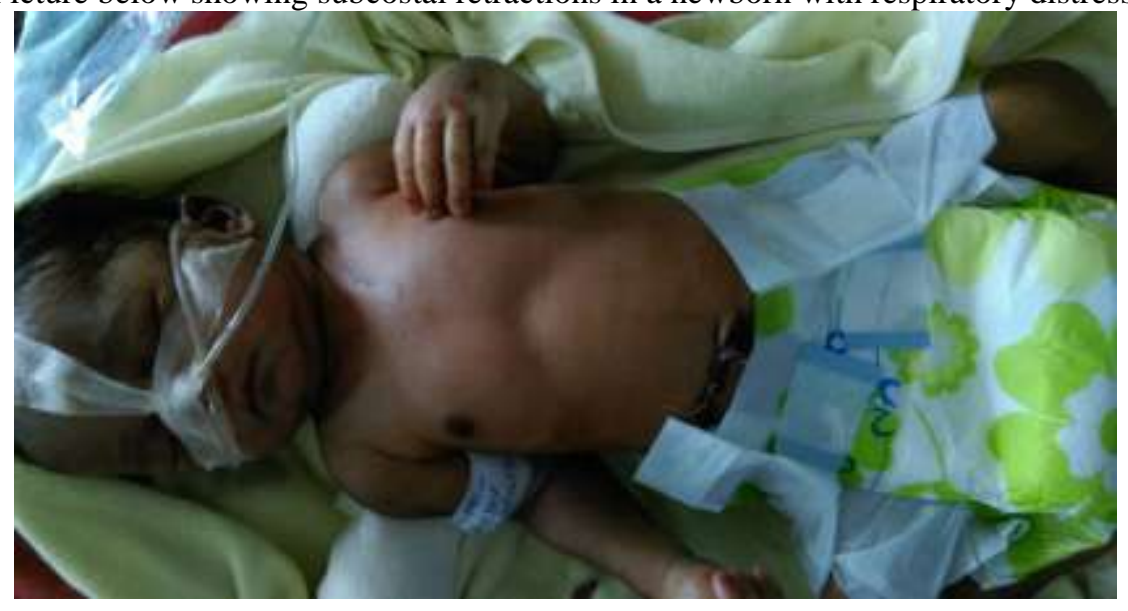




\section{Discussion}

In the study done among 100 patients presenting with features of respiratory distress in NICU,maximum cases were that of infections(32\%) followed by Transient Tachypnea Of Newborn(22\%).Respiratory Distress was more common among Males(68\%) vs Females(32\%).Pulmonary causes $(64 \%)$ were more compared to Non-Pulmonary causes $(36 \%)$ leading to Respiratory Distress. Respiratory Distress was less seen among Term( 38\%) babies vs Pre-Term(62\%) babies.35 cases were present in the weight group of 2.1KG-2.5KG.Hyaline Membrane Disease was most common among the gestational age group 28 weeks - 31 weeks.L.S.C.S mode of delivery lead to lesser respiratory distress when compared to vaginal mode of delivery. Both Hyaline Membrane Disease(40\%) and Infections(40\%) had the highest rate of deaths during the study. Among other causes list included conditions like: diaphragmatic hernia, trachea-oesophageal fistula, pneumothorax following forceful AMBU ventilation,hypocalcemia,hyperglycemia. According to CHRISTIAN L.HERMANSEN AND KEVIN N.LORAH et al.(2007) ${ }^{6}$ Transient Tachypnea of the newborn is the most common cause of neonatal respiratory distress, constituting more than $40 \%$ of cases. But in our region where most of the delivery are normal vaginal delivery and a fair number of cases were with intrauterine growth retardation so incidence is less.

\section{Conclusion}

Respiratory Distress is one of the most common cause of NICU admission and mortality among the newborns. Infections/Sepsis is the leading cause of respiratory distress especially among late pre-term or term baby while Hyaline Membrane Disease is most common cause of respiratory distress among pre-term babies.vaginal mode of delivery is less commonly associated with respiratory distress. Male babies are more prone to develop respiratory distress.

\section{Reference}

[1]. Manual Of Neonatal Care( $7^{\text {th }}$ edition)-John P Cloherty[pg-407-410].

[2]. Banerjee CK,Mahajan,Narang AS-Indian Paediatrics No.8.

[3]. Nelson Textbook Of Pediatrics $19^{\text {th }}$ edition(pg 581-590).

[4]. Caffey J:Text book of Pediatrics X-ray diagnosis $10^{\text {th }}$ edition (pg 1995 ).

[5]. AIIMS NICU protocols.

[6]. Christian L.Hermansen And Kevin N.Lorah Et Al(2007). 